\title{
miR-451a induced apoptosis of Philadelphia chromosome-positive acute lymphoblastic leukemia cells by targeting IL-6R
}

\author{
T. JIANG ${ }^{1}$, J. CHEN 1 , X. B. HUANG ${ }^{1}$, Y. X. LI' ${ }^{2}$ L. ZHONG ${ }^{2, *}$ \\ ${ }^{1}$ Department of Hematology, Sichuan Academy of Medical Sciences and Sichuan Provincial People's Hospital, Chengdu, Sichuan, China; \\ ${ }^{2}$ Department of Clinical Laboratory, Sichuan Academy of Medical Sciences and Sichuan Provincial People's Hospital, Chengdu, Sichuan, China
}

${ }^{*}$ Correspondence: zhonglingr@126.com

Received January 21, 2018 / Accepted May 2, 2018

\begin{abstract}
Philadelphia chromosome positive $(\mathrm{Ph}+)$ acute lymphoblastic leukemia (ALL) is the most fatal leukemia due to the $\mathrm{BCR} / \mathrm{ABL}$ fusion protein. This fusion protein can induce interleukin 6 (IL-6) expression in leukemia stem cells (LSCs) which sustain stemness by binding IL-6R and activating the Janus kinase (JAK)/signal transducer and activator of the transcription (STAT) pathway. IL-6R was one of the targets of miR-451a down-regulated in LSCs by BCR/ABL. We investigated the relationship between miR-451a, IL-6R, and BCR/ABL in Ph+ ALL and created a strategy to treat this disease. The expression levels of miR-451a and BCR/ABL of Ph+ ALL patients were examined by real-time quantitative polymerase chain reaction (RT-qPCR) and serum IL-6 was tested by enzyme-linked immunosorbent assay. Ph+ ALL cell line SUP-B15 and $\mathrm{Ph}-\mathrm{ALL}$ cell line Nalm-6 were treated with miR-451a mimic and inhibitor, respectively; proliferation rate was assessed by CCK-8, apoptosis rate was tested by Annexin/PI and the expression levels of Bcl-XL, Bax, cyclin D2 and c-myc were examined by qPCR and western blot (WB). The levels of STAT3, p-STAT3, JAK2, and p-JAK2 were tested by WB. We found that BCR/ABL was inversely related to miR-451a and positively related to IL-6 in Ph+ ALL. MiR-451a inhibited the proliferation of SUP-B15 through the apoptosis pathway. The oncogene c-myc was down-regulated by miR-451a. We confirmed that miR-451a could target IL-6R and inhibit activation of JAK and STAT3. In conclusion, miR-451a is down regulated in $\mathrm{Ph}+\mathrm{ALL}$ and increasing the expression levels of miR-451a in leukemia cells can increase the potential of curing this disease.
\end{abstract}

Key words: Ph+ ALL, IL-6, miR-451a, signaling pathway, apoptosis

Philadelphia chromosome positive $(\mathrm{Ph}+)$ acute lymphoblastic leukemia (ALL) is the most fatal leukemia despite the fact that tyrosine kinase inhibitors (TKI) have improved the prognosis of this vital disease $[1,2]$. One of the main reasons leukemia is difficult to cure is that the survival of leukemia stem cells (LSCs) in the protective areas of the bone marrow (BM) constantly lead to relapse [3]. Although the BCR/ABL fusion protein is the main reason for the poor outcome in patients with $\mathrm{Ph}+\mathrm{ALL}$, it is unable to confer the long-term stemness of committed B-lymphoid progenitors [4]. In chronic myeloid leukemia (CML), BCR/ABL fusion protein induces the expression of interleukin 6 (IL-6), a pleiotropic cytokine and creates a hospitable micro-environment for leukemia to develop and sustain the stemness of LSCs [5]. While the BCR/ABL fusion gene is the characteristic marker of $\mathrm{Ph}+\mathrm{ALL}$, the relationship between IL-6 and BCR/ABL fusion gene in $\mathrm{Ph}+\mathrm{ALL}$ needs further investigation.
IL-6 initiates its action by binding to its receptor (IL-6R) and then triggering activation of the Janus kinase (JAK)/ signal transducer and activator of transcription 3 (STAT3) signaling pathway $[6,7]$. Activation of this pathway involves activation of the cytokine receptor, subsequent tyrosine phosphorylation of intracellular JAKs and recruitment and phosphorylation of STATs [8]. Phosphorylated STAT proteins dimerize, translocate to the nucleus and initiate target gene transcription [9]. Aberrant activation of JAK/ STAT3 signaling, in particular STAT3, participates in the initiation, development and progression of human cancers via induction of STAT3 down-stream genes that encode anti-apoptotic proteins, cell cycle regulators, and proliferation factors such as Bcl- $\mathrm{X}_{\mathrm{L}}$, Cyclin D2 and c-myc [10, 11]. They play an important role in cell proliferation and apoptosis and participate in the pathogenesis of hematological malignancies [12]. 
MicroRNAs are small, single stranded RNA molecules that regulate gene expression through translational repression or mRNA cleavage, and these play an important role in cancer development. Bioinformatic analysis revealed that IL-6R was among the predicted targets of miR-451a [13]. MiR-451a can inhibit the proliferation of several tumor cells, including lung adenocarcinoma and renal cell carcinoma $[14,15]$. In chronic myeloid leukemia (CML), miR-451a is inversely correlated with BCR-ABL transcript levels and its low expression is related to imatinib resistance [16]. This study investigates the effects and mechanism of miR-451a on the $\mathrm{Ph}+\mathrm{ALL}$ cell line.

\section{Materials and methods}

Cell lines and cell culture. Human Ph+ ALL cell line SUP-B15 (ATCC ${ }^{\oplus}$ Number: CRL-1929 ${ }^{\mathrm{m}}$ ) and Ph- ALL cell line Nalm-6 (ATCC ${ }^{\circledR}$ Number: CRL-3273 ${ }^{\text {max }}$ ) were cultured in complete RPMI 1640 medium containing 10\% FBS (Gibco, Burlington, Canada). All experiments were performed on the cells at passages 3-8 with 80-90\% confluence.

Patient samples. We collected BM samples and peripheral blood samples from $46 \mathrm{Ph}+\mathrm{ALL}$ patients. This study was approved by the institutional review board of our hospital and was conducted according to the Declaration of Helsinki. All patients provided written consent. BM mononuclear cells (BM-MNCs) were isolated by Percoll discontinuous density gradient centrifugation.

Real-time PCR. Total RNA was extracted using TriPure isolation reagent (Roche) and then reverse transcribed to cDNA with first-strand cDNA synthesis kit (Roche) using random primers. We used SYBR Premix Ex Taq (Takara Bio) with specific primers to perform real-time PCR (q-PCR) for the gene expression analysis of BCR/ABL, IL-6, Bcl-X, Bax, cyclin D2, and c-myc. We used the $2^{-\Delta \Delta C t}$ method for data analysis and $\beta_{2}$ microglobin as the RNA integrity control. MiR-451a was quantified by qPCR using the SYBR Green PCR Kit (Ribo, China) and U6 was the internal reference for miR-451a quantification.

IL-6 measurements. IL-6 was measured by commercially available enzyme-linked immunosorbent assays (Abnova, USA) according to the manufacturer's recommendations. Standards and samples were measured in duplicate and all values were expressed as the mean of the two determinations. The concentration was determined by standard curve and the lower level of assay sensitivity was $<2 \mathrm{pg} / \mathrm{ml}$. All analyses were performed at least three times for each individual cellstimulation assay.

Cell viability assay. Cell viability assay was performed using the CCK-8 Assay Kit (Dojindo Molecular Technologies) according to the manufacturer's protocol. Briefly, cells were seeded into 96-well plates at a density of $2 \times 10^{3} /$ well and incubated in different groups for 48 hours. CCK- 8 solution $(10 \mu \mathrm{l} /$ well $)$ was added to each well and the plate was incubated at $37^{\circ} \mathrm{C}$ for another hour. The absorbance was measured by microplate reader (BioTek) at a wavelength of $450 \mathrm{~nm}$. The cell viability was normalized by control cells; where the value of control cells equals 1 .

Cell proliferation assay. Cell proliferation was determined by BrdU Assay Kit (Roche). The cells were prepared in a black 96-well MP to final volume of $100 \mu \mathrm{l} /$ well and incubated at $37^{\circ} \mathrm{C}$ in a humidified atmosphere for 48 hours. Then, a $10 \mu \mathrm{l} /$ well BrdU labelling solution (final concentration $10 \mu \mathrm{M} \mathrm{BrdU}$ ) was added, and the cells were further incubated for a further 2 hours at $37^{\circ} \mathrm{C}$. After removing the labelling medium by tapping off, the cells were fixed by adding a $200 \mu \mathrm{l} /$ well FixDenat solution and incubated for 30 minutes. The cells were incubated with anti-BrdU-POD solution for 2 hours. After washing the wells three times, a substrate reaction was performed. The light emission of the samples was measured using a microplate reader (BioTek).

Cell apoptosis assay. Cell apoptosis was determined using an Annexin V-FITC staining kit (Roche) following the manufacturer's instructions. After the treatment, cells were collected and washed with PBS, gently re-suspended in the binding buffer and incubated with Annexin V-FITC and/or propidium iodide (PI) for 30 minutes in the dark. Apoptotic cells were analyzed via a flow cytometer.

IL-6R rescue. IL-6R-over-expressing vectors were constructed by OriGene (USA). IL-6R-over-expressing plasmids contain either wild-type (wt) or mutant (mut) transcripts with mutations in miR-451a binding sites. The experimental groups were divided into control, IL-6R wt plus miR-451a mimic, IL-6Rwt plus mimic control, IL-6Rmut plus miR-451a and IL-6Rmut plus mimic control.

Western blotting. Proteins were extracted from SUP-B15 with a lysis buffer containing PMSF and the concentration was determined by BCA Protein Assay Kit (Beyotime Biotechnology). Protein extracts were run on SDS/PAGE and transferred to polyvinylidene difluoride membranes. After blockage with $4 \%$ non-fat milk, the membranes were probed with the indicated primary antibodies against Bcl- $\mathrm{X}_{\mathrm{L}}, \mathrm{Bax}$, cyclin D2, c-myc, JAK ${ }_{2}, \mathrm{p}-\mathrm{JAK}_{2}, \mathrm{STAT}_{3}$, and p-STAT 3 (cat. ab178844, ab32503, ab220275, ab32072, ab108596, ab68153, ab76315, abcam) overnight at $4{ }^{\circ} \mathrm{C}$. The membranes were then washed and incubated with horseradish peroxidase (HRP)-conjugated secondary antibody at 1:2000 dilution for 1 hour. The proteins were visualized by enhanced chemiluminescence (ECL) reagents in the Molecular Imager Gel Doc XR System (Bio-Rad).

Luciferase reporter assay. Human IL-6R 3'-UTR with a mutation of the miR-451a seed sequence (mut) was amplified and inserted between the restrictive sites Xho I and Not I of firefly and Renilla luciferase reporter vector pmiR-RB-REPORT (RiboBio Co., Ltd). The $293 \mathrm{~T}$ cells were plated at $1 \times 10^{5} /$ well on 24 -well plates and transfected with 30 ng luciferase reporter vectors. Twenty-four hours posttransfection, firefly and Renilla luciferase activities were consecutively measured according to the dual-luciferase assay manual (Promega). The Renilla luciferase signal was 
normalized to the firefly luciferase signal for each individual analysis.

Statistical analysis. Results are expressed as the means \pm SD from three independent experiments. Statistical analysis was performed using SPSS version 19.0 (SPSS Inc.), and comparisons between two groups were measured by Student's t-test. A two-sided $\mathrm{p}$-value of $<0.05$ was considered statistically significant.

\section{Results}

$\mathrm{BCR} / \mathrm{ABL}$ was inversely related to miR-4p51a and positively related to IL-6 in Ph+ ALL patients. To investigate the relationship between $\mathrm{BCR} / \mathrm{ABL}$ and $\mathrm{miR}-451 \mathrm{a}$, we tested the expression levels of $\mathrm{BCR} / \mathrm{ABL}$ and miR-451a in $\mathrm{BM}-\mathrm{MNCs}$ of $\mathrm{Ph}+\mathrm{ALL}$ patients; we found that $\mathrm{BCR} / \mathrm{ABL}$ mRNA was inversely related to miR-451a $(r=-0.88 ; \mathrm{p}<0.05)$ (Figure 1A). To investigate the relationship between BCR/ ABL and IL-6, we tested the expression level of BCR/ABL in BM-MNCs and concentration of IL-6 in the serum of Ph+ ALL patients; we found that BCR/ABL mRNA was positively related to serum IL-6 concentration $(r=0.87 ; \mathrm{p}<0.05)$ (Figure 1B).

Differences in the expression levels of miR-451a and IL-6 between SUP-B15 and Nalm-6. We tested the expression levels of miR-451a and IL-6 in Ph+ ALL cell line SUP-B15 and Ph-ALL cell line Nalm-6. The relative expression level of miR-451a was $14.09 \pm 3.82$ in SUP-B15 and
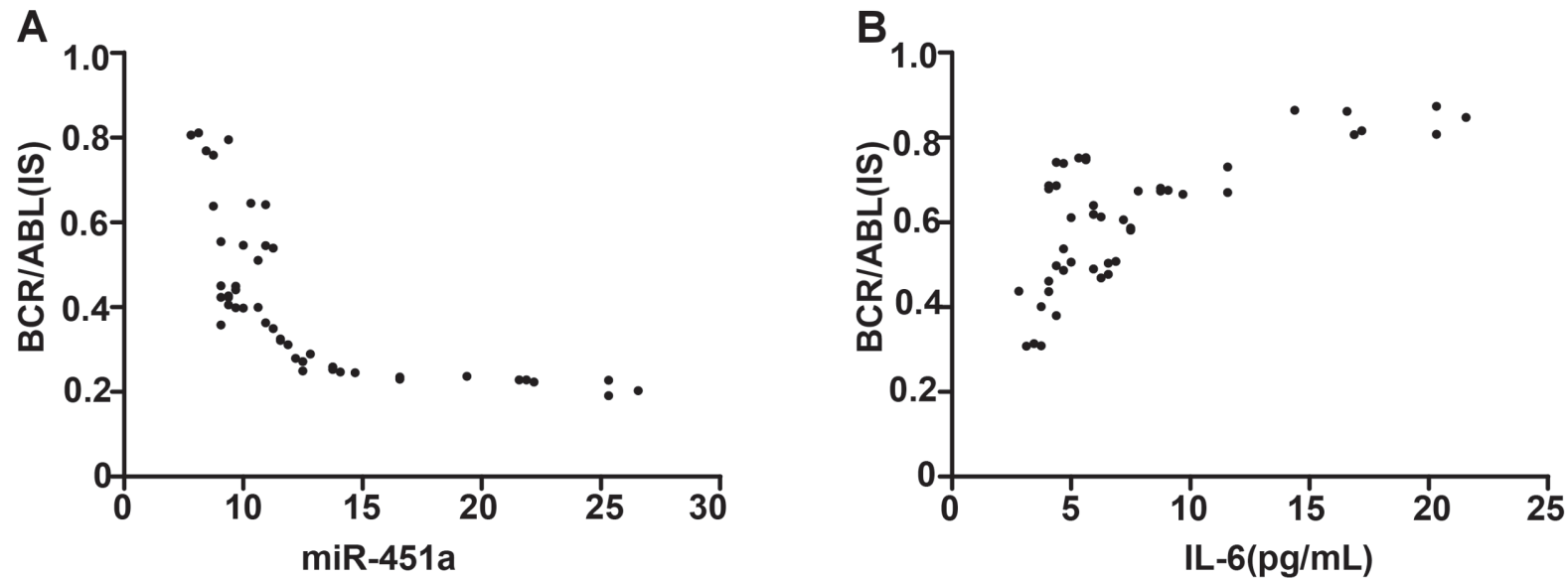

Figure 1. Relationship of BCR/ABL and miR-451a, IL-6. A) the expression levels of BCR/ABL were inversely related with miR-451a; the $x$-axis indicates the relative expression of miR-451a and the $y$-axis indicates the IS value of BCR/ABL; $B$ ) the expression levels of BCR/ABL were positively related with IL-6; the $x$-axis indicates the serum concentration of IL- 6 and the $y$ axis indicates the IS value of BCR/ABL.

A

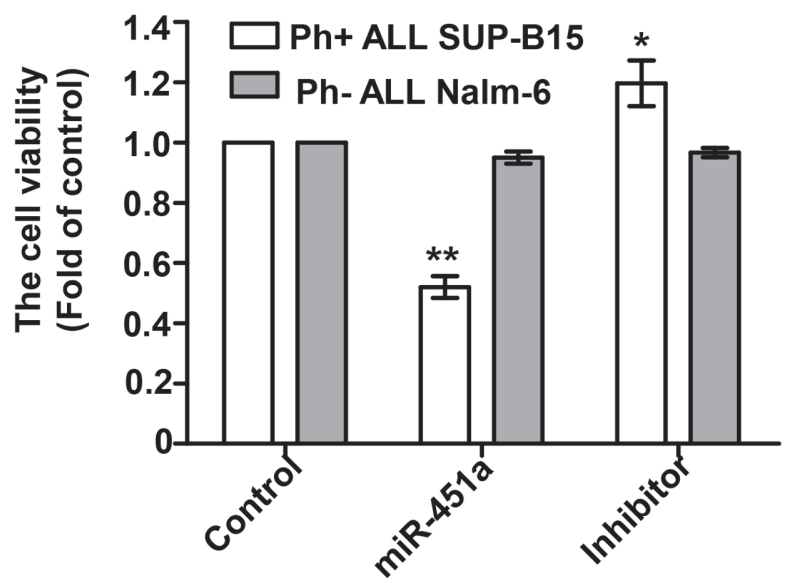

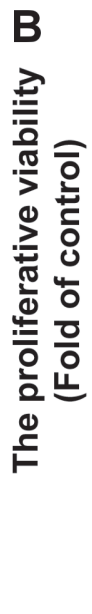

Figure 2. miR-451a inhibits Ph+ ALL. A) miR-451a mimic inhibited the cell viability of Ph+ ALL cell line SUP-B15 but not Ph- ALL cell line Nalm-6; miR-451a inhibitor slightly increased the cell viability of SUP-B15; B): The proliferative ability of SUP-B15 and Nalm-6 were tested by BrdU assay after miR-451a mimic and inhibitor treatment, respectively. The proliferation rate of SUP-B15 was significantly decreased after miR-451a mimic treatment, the proliferation rate of Nalm-6 was not affected, and the proliferation rate of SUP-B15 was slightly increased after miR-451a inhibitor treatment; ${ }^{*}$ : $\mathbf{p}<\mathbf{0 . 0 5} ;{ }^{* *}: \mathbf{p}<\mathbf{0 . 0 1}$. 
$194.76 \pm 36.89$ in Nalm-6 ( $\mathrm{p}=0.0002)$. The relative expression of IL- 6 was highest in SUP-B15 $(98.75 \pm 21.46)$ and lowest in Nalm-6 (25.67 \pm 8.24$)$. We tested the IL-6 levels in culture supernatants and found that IL- 6 was significantly higher in the culture supernatant of SUP-B15 $(43.62 \pm 4.54 \mathrm{pg} / \mathrm{ml})$ than in Nalm-6 (11.48 $\pm 3.81 \mathrm{pg} / \mathrm{ml})$.

MiR-451a mimics inhibiting cell proliferation of SUP-B15 through the apoptosis pathway. To test if miR-451a could inhibit cell proliferation of $\mathrm{Ph}+\mathrm{ALL}$, we treated both cell lines with miR-451a mimic and inhibitor. The cell viability of SUP-B15 was significantly decreased after miR-451a mimic treatment $(52.01 \% \pm 2.14 \%$; $\mathrm{p}=0.00003)$ (Figure 2A) and increased after miR-451a inhibitor treatment (119.73\% $44.46 \% ; \mathrm{p}=0.014)$, while Nalm-6 was unaffected.

MiR-451a mimic could inhibit the cell proliferation ability of SUP-B15 $(44.32 \% \pm 2.64 \%$; $\mathrm{p}=0.00006)$ but not Nalm-6 (Figure 2b), while the miR-451a inhibitor increased cell proliferation ability of SUP-B15 (110.01\% $\pm 8.21 \%$; $\mathrm{p}=0.45)$ but not Nalm-6 (Figure 2B).

We further tested the apoptotic rate by Annexin/PI staining and found that the apoptotic rate of SUP-B15 was significantly increased after miR-451a treatment (39.66\%; $\mathrm{p}=0.00001$ ), but decreased after miR-451a inhibitor treat- ment $(0.01 \%)$ (Figure 3). Nalm-6 remained unchanged after both treatments. We tested the apoptotic and anti-apoptotic proteins of SUP-B15 24 hours after miR-451a mimic and inhibitor treatment and found that the relative expression of anti-apoptotic protein Bcl-X decreased significantly after miR-451a mimic treatment $(0.53 \pm 0.05$ folds to control $)$ and increased after miR-451a inhibitor treatment $(1.96 \pm 0.10$ folds to control) (Figure 4A). In contrast, the relative expression of apoptotic protein Bax increased significantly after miR-451a mimic treatment $(3.85 \pm 0.12$ folds to control) and decreased after miR-451a inhibitor treatment $(0.69 \pm 0.06$ folds to control) (Figure 4A). The protein levels of Bcl- $\mathrm{X}_{\mathrm{L}}$ and Bax also changed accordingly (Figures $4 \mathrm{~B}$ and 5A).

MiR-451a inhibits the expression of oncogenic proteins in SUP-B15. Cyclin D2 is consistently over-expressed in Ph+ ALL and enables leukemia cells to bypass normal controls over the mitotic cell cycle and to achieve a deregulated state of proliferation [17]. C-myc is an important down-stream mediator of pre-BCR signaling in B cell ALL [18]. We tested the expression of both genes in SUP-B15 after miR-451a mimic and inhibitor treatment and found that cyclin D2 decreased to $0.49 \pm 0.04$ folds compared to controls after mimic treatment and increased to $2.12 \pm 0.13$ folds after
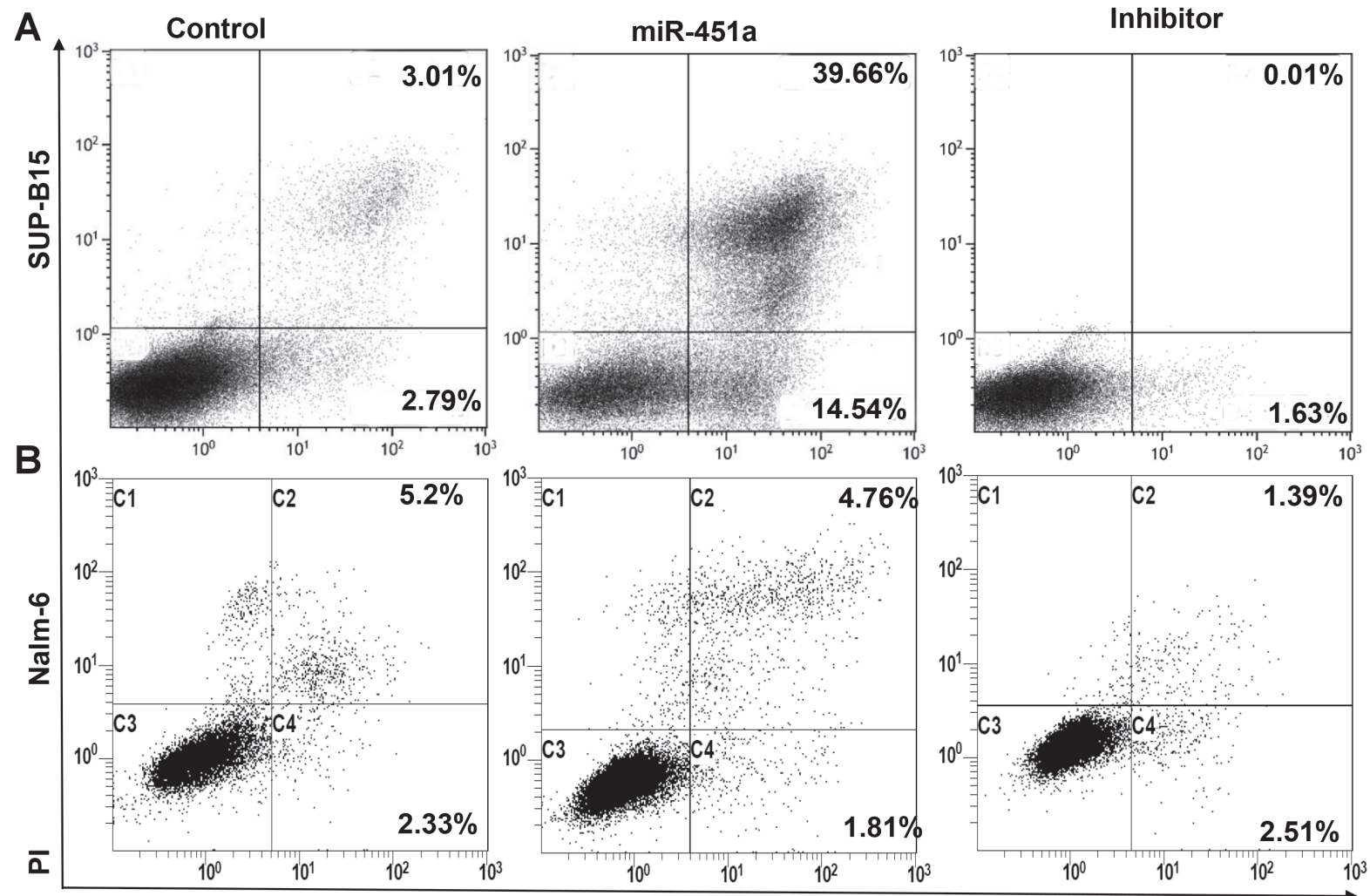

Annexin-V FITC

Figure 3. miR-451a induces apoptosis of Ph+ ALL. A) The apoptosis rate of SUP-B15 increased after miR-451a mimic and inhibitor; B) The apoptosis rate of Nalm-6 was not affected after miR-451 a mimic treatment. 

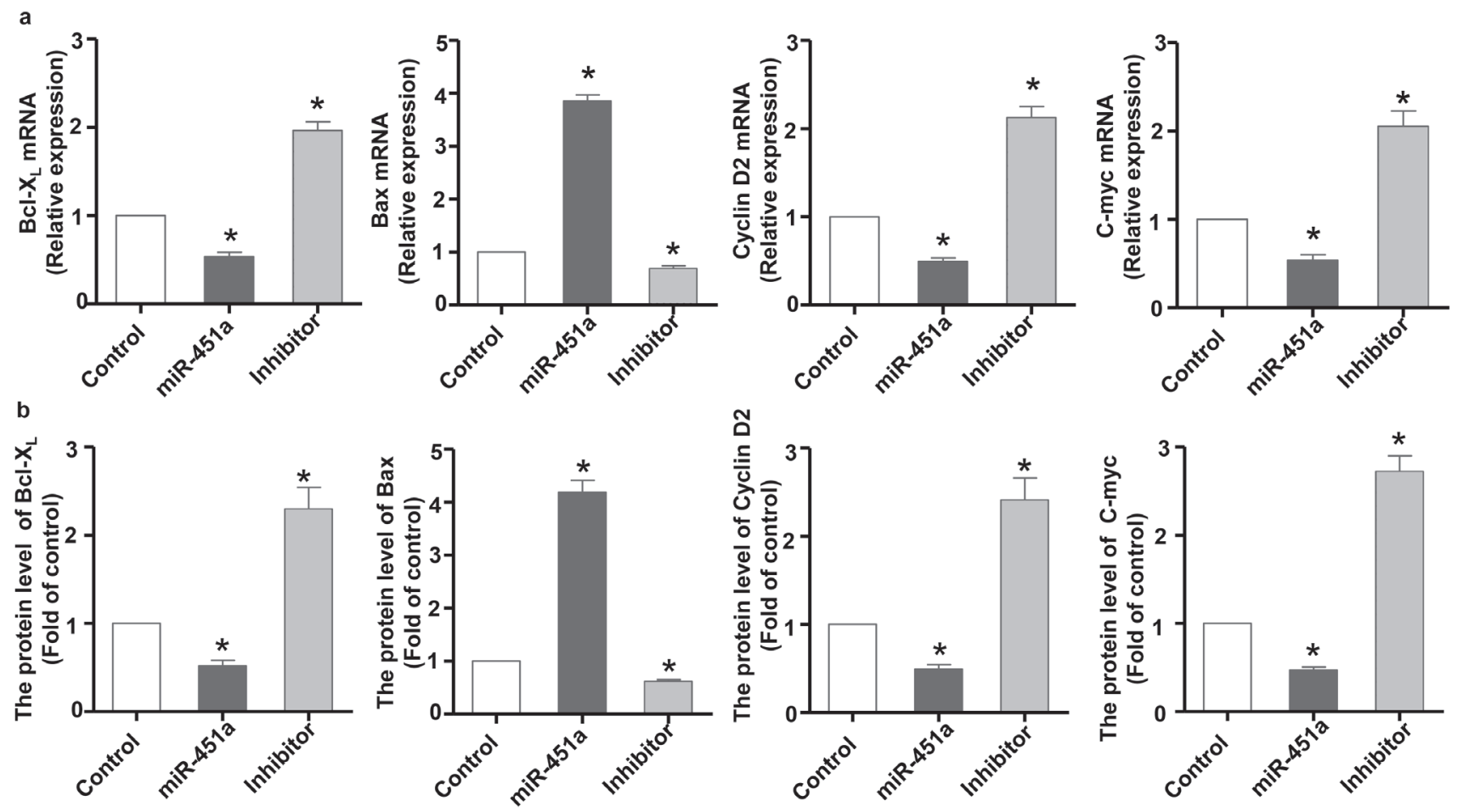

Figure 4. Gene expression change in SUP-B15 after miR-451a treatment. A) The mRNA levels of Bcl- $X_{L}$, Bax, cyclin D2, and c-myc of SUP-B15 after miR-451a mimic and inhibitor treatment, respectively; B) The protein levels of Bcl- $X_{L}$, Bax, cyclin D2, and c-myc of SUP-B15 after miR-451a mimic and inhibitor treatment, respectively.

inhibitor treatment, and c-myc decreased to $0.54 \pm 0.07$ folds after mimic treatment and increased to $2.05 \pm 0.17$ folds after inhibitor treatment (Figure $4 \mathrm{~A})$. The protein levels of both genes changed accordingly (Figures $4 \mathrm{~B}$ and $5 \mathrm{~A}$ ).

Validation of IL-6R as miR-451a target. IL-6 binds to IL-6R and subsequently activates the JAK/STAT3 signaling pathway to initiate further biologic function. To examine whether miR-451a is essential to IL-6 in regulating SUP-B15 proliferation, we tested the level of total and phosphorylated JAK2 and STAT3. We found that miR-451a reduced the levels of phosphorylated JAK2 and STAT3 (p-JAK2 and p-STAT3) without affecting the levels of total JAK2 and STAT3 (Figure 5B).

IL-6R is predicted to be a miR-451a target in silico [13]. We used the 3'-UTR luciferase reporter assays to validate direct repression of IL-6R by miR-451a. Luciferase activity in cells containing wt 3'-UTR of IL-6R was reduced in response to miR-451a, while luciferase activity was not affected in cells containing mut 3'-UTR of IL-6R in response to miR-451a. Furthermore, scrambled mimic control made no changes in either cell. These results indicate that miR-451a directly represses IL-6R by targeting its 3'-UTR (Figure 6).

We also performed an IL-6R rescue experiment. IL-6Rover-expressing plasmids contained either wt transcripts or mut transcripts with mutations in the miR-451a-binding sites. We set up five groups: control group, miR-451a mimic plus IL-6Rwt group, IL-6Rwt plus miR-451a control (scrambled mimic control) group, IL-6Rmut plus miR-451a mimic group and the IL-6Rmut plus miR-451a control group. We found that either IL-6Rwt over-expression or IL-6Rmut overexpression causes over-proliferation of SUP-B15 and that miR-451a inhibits SUP-B15 with IL-6Rwt over-expression but not with IL-6Rmut over-expression (Figure 7A). The apoptosis rate of SUP-B15 cells in the miR-451a mimic plus IL-6Rwt group was increased, while that in all other groups remained unchanged (Figures 7B-F).

\section{Discussion}

In this study, we found that $\mathrm{BCR} / \mathrm{ABL}$ transcripts were inversely related to the expression level of miR-451a in $\mathrm{Ph}+$ ALL and positively related to serum IL- 6 concentration. We then demonstrated that miR-451a could inhibit the proliferation of the $\mathrm{Ph}+\mathrm{ALL}$ cell line through the apoptosis pathway, and observed that miR-451a could up-regulate intrinsic apoptosis protein $\mathrm{Bax}$ and down-regulate anti-apoptosis protein Bcl- $\mathrm{X}_{\mathrm{L}}$ and oncogenic protein cyclin D2 and c-myc. Most importantly, we confirmed that miR-451a can inhibit the IL-6R/JAK/STAT3 pathway by direct repression of IL-6R and down-stream p-JAK and p-STAT3. 
A

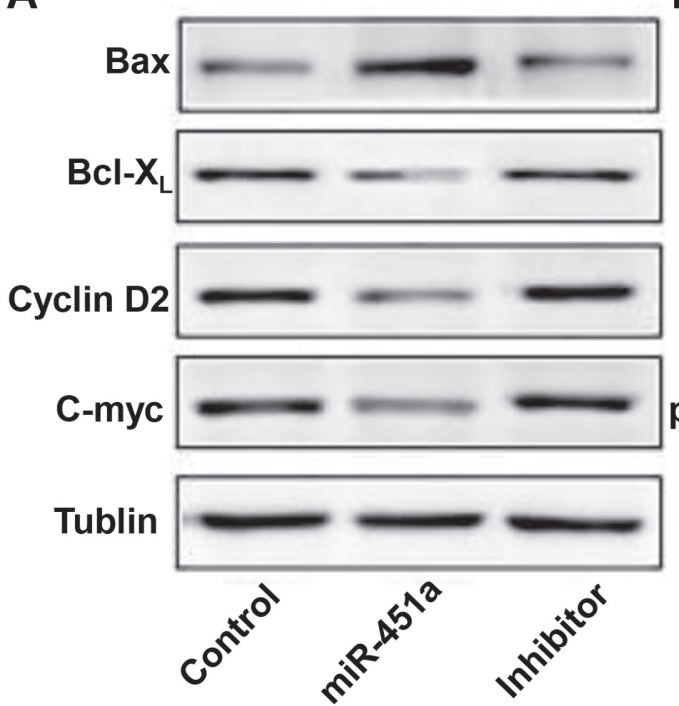

B

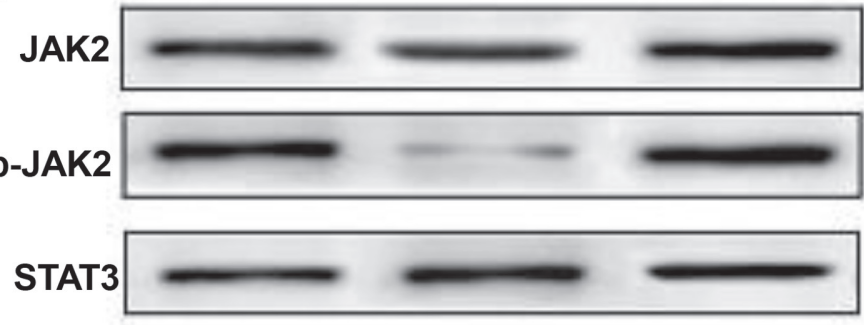

p-STAT3

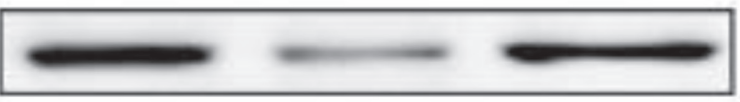

GAPDH

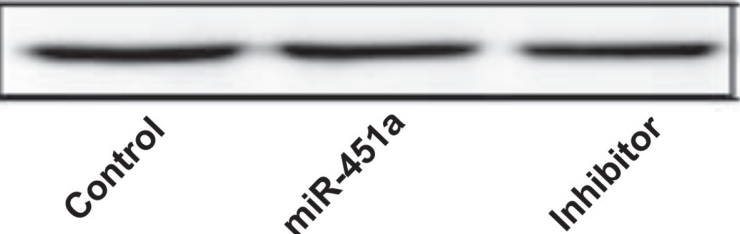

Figure 5. Signal pathway change of SUP-B15 after miR-451a treatment. A) Western blot results of Bcl-X, Bax, cyclin D2, and c-myc of SUP-B15 after miR-451a mimic and inhibitor treatment; B) Western blot of total JAK2 and STAT3 and phosphorylated JAK2 and STAT3 of SUP-B15 after miR-451a mimic and inhibitor treatment.

A

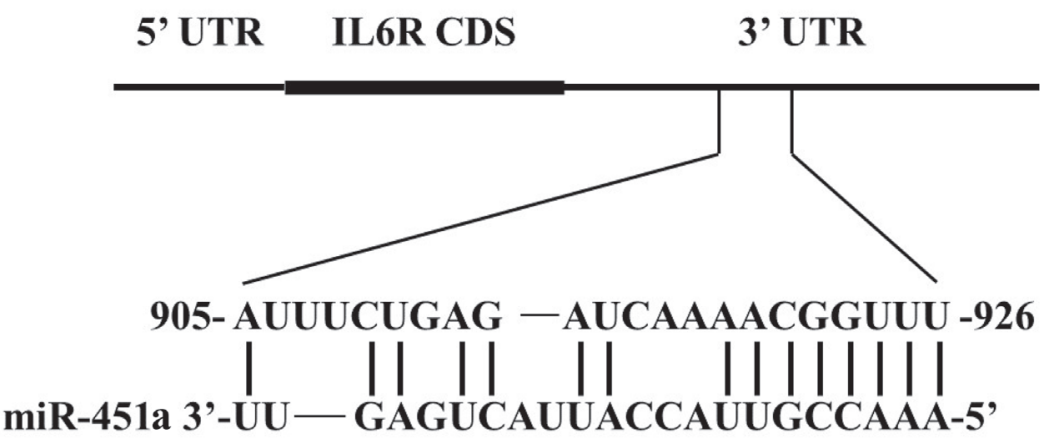

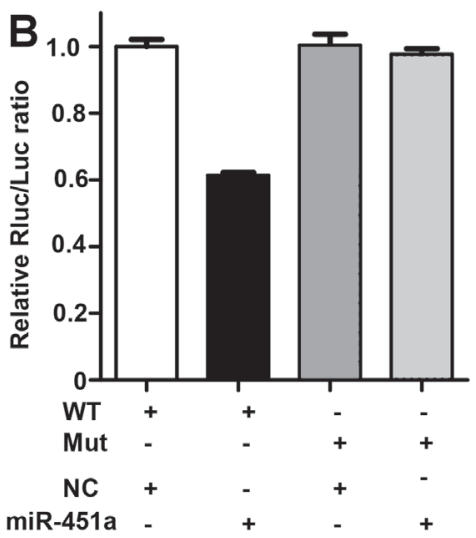

Figure 6. Validation of IL-6R as an miR-451a target. A) Binding site and sequence of miR-451a targeting IL-6R; B) Relative Rluc/Luc ratio analysis showed that hsa-miR-451a could significantly reduce the reported fluorescence of IL-6R wild-type vectors. After binding sites were mutated, the reported fluorescence in the vector was nearly restored to control levels. Compared to the control group, $\mathrm{p}<0.05$.

$\mathrm{Ph}+\mathrm{ALL}$ is one of the most virulent hematologic malignancies, with a 5 -year overall survival rate of $<5 \%$ without allogeneic hematologic stem cell transplantation (alloHSCT) [19]. TKI have significantly improved the prognosis of Ph+ ALL [20]; however, allo-HSCT is still needed for most of the patients $[21,20]$. One of the main reasons for leukemia to relapse is that LSCs are difficult to eradicate. LSCs are sheltered in the BM niche and are protected by the tumor micro-environment, making them capable of escaping the killing effects of chemotherapy drugs and immune clearance. IL- 6 has a critical role in the pro-tumorigenic inflammatory environment in CML pathogenesis and some other cancers $[5,22]$. IL- 6 establishes a paracrine feedback loop that re-directs normal and leukemic multipotent progenitor's differentiation potential towards the myeloid lineage and contributes to CML development [5]. Autocrine and paracrine IL- 6 can initiate immunosuppressive effects through the IL-6R/JAK/STAT pathway, and this could further increase the survival of leukemia cells. This study found that the Ph+ ALL cell line secrets higher levels of IL- 6 than the Ph- ALL cell line. This may partly explain why Ph+ ALL is difficult to cure and why TKI could significantly improve patient outcomes.

IL-6R was among the predicted targets of miR-451a [13] and this was confirmed by luciferase reporter assay. The expression level of miR-451a was regulated by $\mathrm{BCR} / \mathrm{ABL}$ 


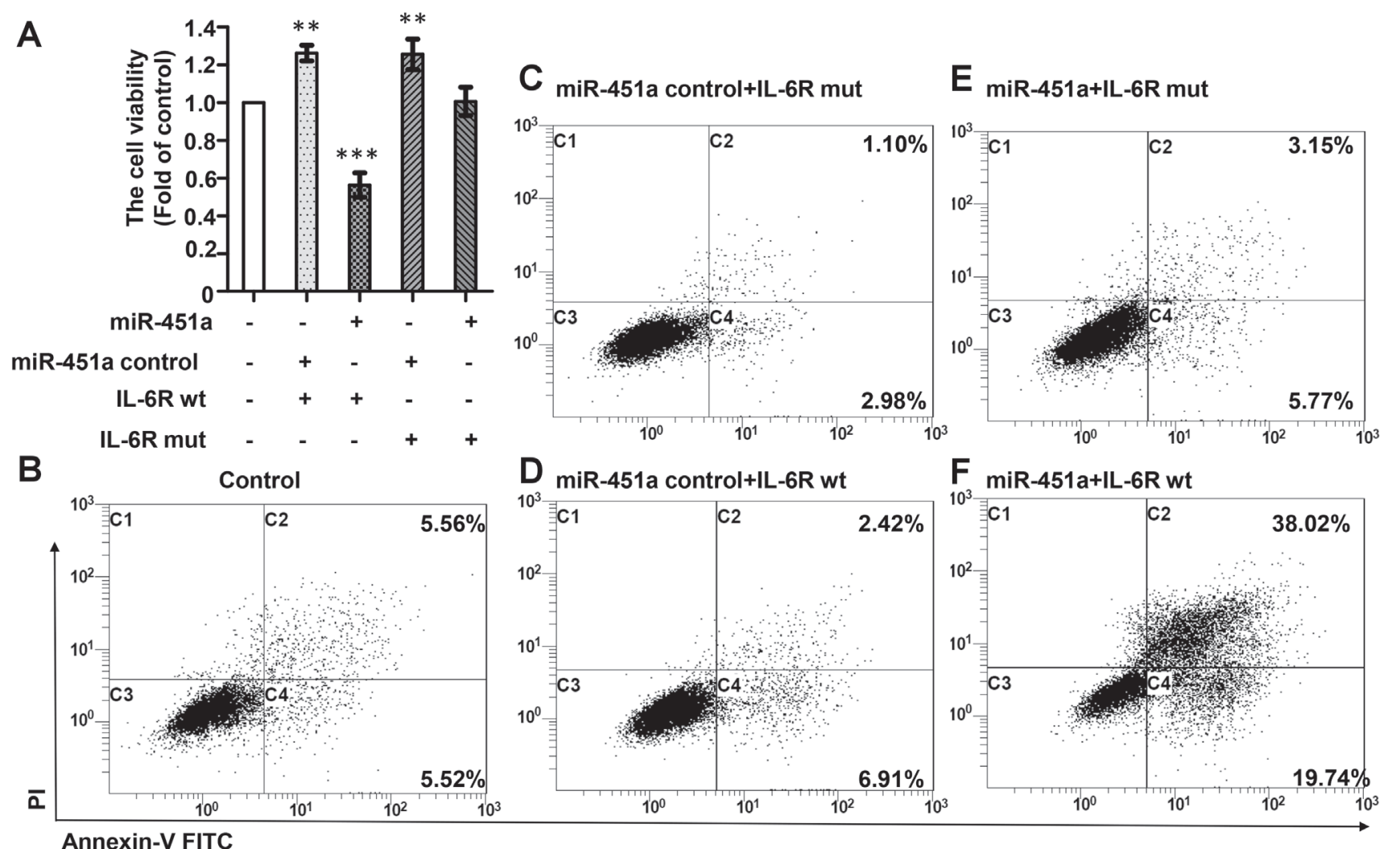

Annexin-V FITC

Figure 7. Rescue of SUP-B15 by IL-6R. A) The cell viabilities of SUP-B15 with either IL-6R wt or IL-6R mut over-expression were slightly increased, the cell viability of SUP-B15 with IL-6R wt over-expression greatly decreased after miR-451a mimic treatment, and the cell viability of SUP-B15 with IL-6R mut over-expression was unaffected after miR-451a mimic treatment. B-F) The apoptosis of SUP-B15 after different treatments. C, D) The apoptosis rate of SUP-B15 with either IL-6R wt or IL-6R mut over-expression was slightly decreased. E) The apoptosis rate of SUP-B15 with IL-6R mut over-expression was unaffected after miR-451a mimic treatment. F) The apoptosis rate of SUP-B15 with IL-6R wt over-expression greatly increased after miR-451a mimic treatment. IL-6R wt: wild-type IL-6R-over-expressing plasmids; IL-6R mut: IL-6R-over-expressing plasmids with mutations in miR-451a-binding sites; miR-451a control: scrambled control sequence; ${ }^{* *}: \mathrm{p}<0.05 ;{ }^{* * *}: \mathrm{p}<0.01$.

fusion gene in CML and was found to be lower in imatinibresistant CML patients $[16,13]$. Ph+ ALL possesses the same fusion gene as CML and it is reasonable to assume that the expression level of miR-451a also decreased, as reported in our study. A possible miR-451/BCR-ABL regulatory loop was suggested in a previous study [16]; however, the link between them is missing. Our results suggested that miR-451a is also down-regulated by BCR/ABL in Ph+ ALL, since miR-451a targets IL-6R which would impair the IL-6 function. By down-regulating miR-451a, Ph+ ALL cells could enhance the activity of IL- 6 and promote cell survival and escape immune clearance.

When we treated Ph+ ALL cell line with miR-451a, the proliferation was significantly inhibited and apoptosis rate increased. The proteins providing survival advantage for $\mathrm{cl}-\mathrm{X}_{\mathrm{L}}, \mathrm{CCDN}$, and c-myc were greatly down-regulated after miR-451a treatment, and the intrinsic apoptosis protein Bax was up-regulated.
Further, IL-6 binds to IL-6R and forms IL-6/IL-6R complex. This complex then associates with and dimerizes the signal-transducing membrane protein gp130. Dimerisation of gp130 rapidly activates the JAK/STAT3 pathway, and the activated STAT3 induces numerous effector genes for cellular proliferation and stem maintenance of tumor cells [12]. Since miR-451a targets IL-6R, as confirmed by luciferase reporter assay, we assume that it suppresses the JAK/ STAT3 pathway in the Ph+ ALL cell line. In this study, we found that p-JAK and p-STAT3 were greatly decreased after miR-451a treatment, while the total protein level of JAK and STAT3 remained the same.

In conclusion, miR-451a is down-regulated by BCR/ABL in $\mathrm{Ph}+\mathrm{ALL}$ to provide survival advantage for leukemia cells through the IL-6/JAK/STAT3 pathway. Therefore, increasing the expression levels of miR-451a in Ph+ ALL leukemia cells of $\mathrm{Ph}+\mathrm{ALL}$ may increase the potential of curing this most virulent $(\mathrm{Ph}+)$ acute lymphoblastic leukemia. 


\section{References}

[1] DEN BOER ML, VAN SLEGTENHORST M, DE MENEZES RX, CHEOK MH, BUIJS-GLADDINES JG et al. A subtype of childhood acute lymphoblastic leukemia with poor treatment outcome: a genome-wide classification study. Lancet Oncol 2009; 10: 125-34. https://doi.org/10.1016/s14702045(08)70339-5

[2] IGWE IJ, YANG D, MERCHANT A, MERIN N, YAGHMOURGetal. The presence of Philadelphia chromosomedoes not confer poor prognosis in adult pre-B acute lymphoblastic leukemia in the tyrosine kinase inhibitor era - a surveillance, epidemiology, and end results database analysis. Br J Haematol 2017; 179: 618-626. https://doi.org/10.1111/bjh.14953

[3] BOYD AL, CAMPBELL CJ, HOPKINS CI, FIEBIG-COMYN A, RUSSELL J et al. Niche displacement of human leukemic stem cells uniquely allows their competitive replacement with healthy HSPCs. J Exp Med 2014; 211: 1925-1935. https://doi.org/10.1084/jem.20140131

[4] LI D, ZHAO X, ZHANG R, JIAO B, LIU P et al. BCR/ABL can promote $\mathrm{CD} 19+$ cell growth but not render them longterm stemness. Stem Cell Investig 2016; 3: 85. https://doi. org/10.21037/sci.2016.11.06

[5] REYNAUD D, PIETRAS E, BARRY-HOLSON K, MIR A, BINNEWIES $M$ et al. IL-6 controls leukemic multipotent progenitor cell fate and contributes to chronic myelogenous leukemia development. Cancer Cell 2011; 20: 661-673. https://doi.org/10.1016/j.ccr.2011.10.012

[6] WANG SW, SUN YM. The IL-6/JAK/STAT3 pathway: potential therapeutic strategies in treating colorectal cancer (Review). Int J Oncol 2014; 44: 1032-1040. https://doi. org/10.3892/ijo.2014.2259

[7] GARBERS C, APARICIO-SIEGMUND S, ROSE-JOHN S. The IL-6/gp130/STAT3 signaling axis: recent advances towards specific inhibition. Curr Opin Immunol 2015; 34: 75-82. https://doi.org/10.1016/j.coi.2015.02.008

[8] CHEN S, DENG G, LI K, ZHENG H, WANG G et al. Interleukin-6 Promotes Proliferation but Inhibits Tenogenic Differentiation via the Janus Kinase/Signal Transducers and Activators of Transcription 3 (JAK/STAT3) Pathway in Tendon-Derived Stem Cells. Med Sci Monit 2018; 24: 15671573. https://doi.org/10.12659/MSM.908802

[9] XIONG S, WANG R, CHEN Q, LUO J, WANG J et al. Cancer-associated fibroblasts promote stem cell-like properties of hepatocellular carcinoma cells through IL-6/STAT3/ Notch signaling. Am J Cancer Res 2018; 8: 302-316.

[10] AGGARWAL BB, KUNNUMAKKARA AB, HARIKUMAR KB, GUPTA SR, THARAKAN ST et al. Signal transducer and activator of transcription-3, inflammation, and cancer: how intimate is the relationship? Ann N Y Acad Sci 2009; 1171: 59-76. https://doi.org/10.1111/j.1749-6632.2009.04911.x

[11] SINGH AK, BHADAURIA AS, KUMAR U, RAJ V, MAURYA $V$ et al. Novel fused oxazepino-indoles (FOIs) attenuate liver carcinogenesis through IL-6/JAK2/STAT3 signaling blockade with strong metabolic regulations. Life Sci 2018; 201: 161-172. https://doi.org/10.1016/j.lfs.2018.02.029
[12] HOSSAIN A, GUMIN J, GAO F, FIGUEROA J, SHINOJIMA N et al. Mesenchymal Stem Cells Isolated From Human Gliomas Increase Proliferation and Maintain Stemness of Glioma Stem Cells Through the IL-6/gp130/STAT3 Pathway. Stem Cells 2015; 33: 2400-2415. https://doi.org/10.1002/ stem.2053

[13] SOLTANI I, DOUZI K, GHARBI H, BENHASSINE I, TEBER $M$ et al. Downregulation of miR-451 in Tunisian chronic myeloid leukemia patients: potential implication in imatinib resistance. Hematology 2017; 22: 201-207. https:// doi.org/10.1080/10245332.2016.1252020

[14] SU Z, NI L, YU W, YU Z, CHEN D et al. MicroRNA-451a is associated with cell proliferation, migration and apoptosis in renal cell carcinoma. Mol Med Rep 2015; 11: 2248-2254. https://doi.org/10.3892/mmr.2014.2957

[15] CHEN Q, HU H, JIAO D, YAN J, XU W et al. miR-126$3 \mathrm{p}$ and miR-451a correlate with clinicopathological features of lung adenocarcinoma: The underlying molecular mechanisms. Oncol Rep 2016; 36: 909-917. https://doi. org/10.3892/or.2016.4854

[16] LOPOTOVA T, ZACKOVA M, KLAMOVA H, MORAVCOVA J. MicroRNA-451 in chronic myeloid leukemia: miR451-BCR-ABL regulatory loop? Leuk Res 2011; 35: 974-977. https://doi.org/10.1016/j.leukres.2011.03.029

[17] DEININGER MW, VIEIRA SA, PARADA Y, BANERJI L, LAM EW et al. Direct relation between BCR-ABL tyrosine kinase activity and cyclin D2 expression in lymphoblasts. Cancer Res 2001; 61: 8005-8013.

[18] KOHRER S, HAVRANEK O, SEYFRIED F, HURTZ C, COFFEY GP et al. Pre-BCR signaling in precursor B-cell acute lymphoblastic leukemia regulates PI3K/AKT, FOXO1 and MYC, and can be targeted by SYK inhibition. Leukemia 2016; 30: 1246-1254. https://doi.org/10.1038/leu.2016.9

[19] LINKER CA, LEVITT LJ, O'DONNELL M, FORMAN SJ, RIES CA. Treatment of adult acute lymphoblastic leukemia with intensive cyclical chemotherapy: a follow-up report. Blood 1991; 78: 2814-2822.

[20] LEE S, KIM YJ, MIN CK, KIM HJ, EOM KS et al. The effect of first-line imatinib interim therapy on the outcome of allogeneic stem cell transplantation in adults with newly diagnosed Philadelphia chromosome-positive acute lymphoblastic leukemia. Blood 2005; 105: 3449-3457. https://doi. org/10.1182/blood-2004-09-3785

[21] RAVANDI F, OTHUS M, O’BRIEN SM, FORMAN SJ, HA CS et al. US Intergroup Study of Chemotherapy Plus Dasatinib and Allogeneic Stem Cell Transplant in Philadelphia Chromosome Positive ALL. Blood Adv 2016; 1: 250-259. https://doi.org/10.1182/bloodadvances.2016001495

[22] TSUKAMOTO H, FUJIEDA K, SENJU S, IKEDA T, OSHIUMI $\mathrm{H}$ et al. Immune-suppressive effects of IL-6 on Tcell-mediated anti-tumor immunity. Cancer Sci 2018; 109: 523-530. https://doi.org/10.1111/cas.13433 